\title{
Muco-epidermoid tumours of the anal canal
}

\author{
B. C. MORSON AND H. VOLKSTÄDT \\ From the Research Department, St. Mark's Hospital, London
}

SYNOPSIS The pathology of 21 cases of muco-epidermoid carcinoma of the anal canal is described These tumours are defined as squamous or transitional cell carcinomas containing areas whichs secrete mucin of undoubted epithelial origin within the clumps of tumour cells. They are founक in that part of the anal canal just above the dentate line where the epithelium is variable in type and represents an embryological transition between rectal and squamous mucous membrane $e_{i}^{\sigma}$ The histogenesis of these tumours is discussed and the opinion given that they arise from the surfacin epithelium of the upper anal canal rather than from the anal glands.

Since Stewart, Foote, and Becker (1945) described muco-epidermoid tumours of the salivary glands growths with a similar histological structure have been described in the oral cavity (Smith, Broadbent, and Zavaleta, 1954), uterine cervix (Hellweg, 1957), urinary bladder (Feyrter, 1956), oesophagus (Azzopardi and Menzies, 1962), and anal canal (Kay, 1954). Berg, Lone, and Stearns (1960) found reports of four cases of muco-epidermoid carcinoma of the anal canal in the literature and added a further nine cases of their own. The object of this paper is to describe the pathology of 21 cases with particular emphasis on their site of origin and histogenesis.

\section{MATERIAL AND METHODS}

One hundred and seventy-eight squamous cell carcinomas of the anus and anal canal seen at St. Mark's Hospital during the 28-year period 1934-1961 inclusive have been reviewed. All histological material was stained by haematoxylin and eosin and the presence of mucin detected either by mucicarmine or alcian blue or by both. By these methods 21 cases of muco-epidermoid carcinoma were found.

In all but three of these cases the rectum, anal canal, and peri-anal skin were removed by synchronous combined excision. The surgical specimens were prepared in the fresh state by cutting along the anterior aspect and stretching them out on a metal frame. The specimens were photographed and an accurate assessment made of the exact site of the primary tumour as well as the extent of local and lymphatic spread. In particular the relationship of the tumour to the dentate line or line of the anal valves was assessed as accurately as possible.

Of the three tumours not treated by excision of the

Received for publication 7 December 1962. rectum one was locally excised and two received radio therapy. In these cases material was available for histo logical study but no assessment of the site of origin of the tumour was attempted.

\section{PATHOLOGY}

INCIDENCE The incidence of muco-epidermoid car cinoma at St. Mark's Hospital is about one in eigh: squamous cell carcinomas of the anus and ana\$ canal. This figure is greater than the one in $1 \%$ reported by Berg et al. (1960) in a review of 126 primary anal cancers from the Memorial Hospital New York. However, the St. Mark's series of 178 . cases includes anal margin as well as anal canats tumours and the latter are about two and a half times more common than the anal margin cancers (Morson, 1960). This means that the incidence of muco-epidermoid carcinoma, when compared witi anal canal cancers only, is about one in six. More over, in about half of the St. Mark's cases mucip was found in relatively small amounts and onl 8 after a careful search.

AGE The age of the patients in this series varief AGE The age of the patients in this series varied from 39 to 73 with an average of about 55 years This is very similar to the age incidence both of adenocarcinoma of the rectum and squamous cef carcinoma of the anal canal (Dukes and Bussey 1958; Morson, 1960).

SEX Nine patients were male and 12 female. In the series of Berg et al. (1960) there were three males an six females. The preponderance of women with squamous cell tumours of the anal canal has beep reported previously (Morson, 1960). 


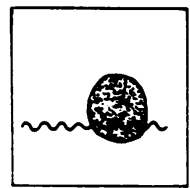

1.

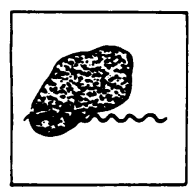

7.

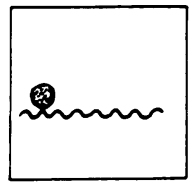

13.

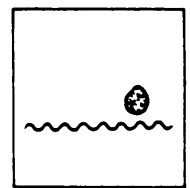

2.

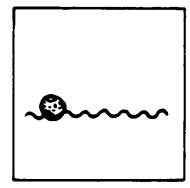

8.

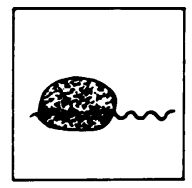

14.

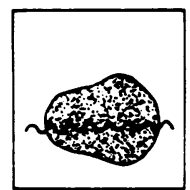

3.

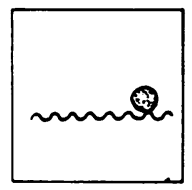

9.

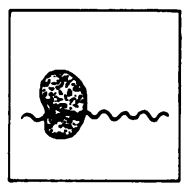

15.

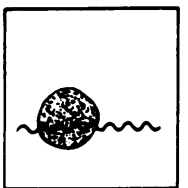

4.

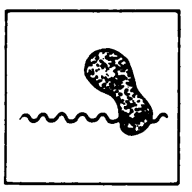

10.

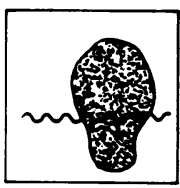

16.

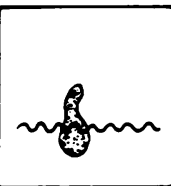

5.

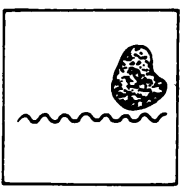

11.

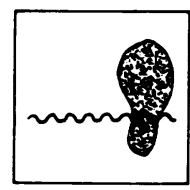

17.

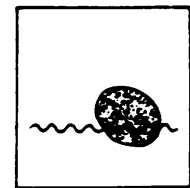

6.

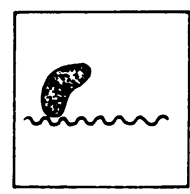

12.

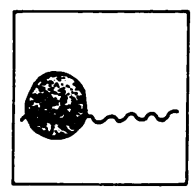

18.

FIG. 1 .

Site of origin

of 18 tumours.

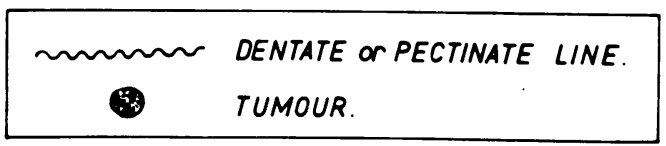

GROSS APPEARANCE AND POSITION OF PRIMARY TUMOURS The tumours in the 18 operation specimens were all flat, ulcerating growths. Some of them were regarded as 'excavating' but none appeared to be covered by intact mucosa or to show any other reason why they were not of surface origin.

The exact position of the tumours relative to the dentate line is given in Figure 1. Twelve tumours were astride but centred above the line while six appeared to be entirely above. The two tumours described by Kay (1954) also appear to be situated in the upper part of the anal canal.

HISTOLOGY For the purposes of this study mucoepidermoid carcinoma is defined as a squamous or transitional cell carcinoma showing secretion of mucin of undoubted epithelial origin within the clumps of tumour cells. Compared with ordinary squamous or transitional cell carcinomas of the anal canal they have a similar histological structure except for the presence of microcysts containing mucin. Muco-epidermoid carcinoma should therefore be regarded only as a variant of squamous cell carcinoma of the anal canal. Adenocarcinomas exhibiting patchy squamous metaplasia and tumours showing contiguous adenocarcinoma and squamous carcinoma have been excluded from this study.

Muco-epidermoid tumours of the anal canal resemble their counterparts in the salivary glands
(Foote and Frazell, 1954), oesophagus, and other sites. Clumps and cords of squamous or transitional cells contain central spaces, or microcysts, filled with strongly staining mucin (Figs. 2 and 3). Sometimes the mucin is present in the form of small pools which give the impression of clumped signet-ring cells (Fig. 4). Single signet-ring cells are also seen, being occasionally found within an epithelial 'pearl' (Fig. 5). In the larger microcysts the mucin is mixed up with the debris of disintegrating tumour cells. In some cases there are very large duct-like spaces within the clumps of tumour containing cell debris which stains only weakly for mucin. Such a duct-like method of growth is quite common in squamous or transitional cell carcinomas of the anal canal in which mucin production cannot be demonstrated. The amount of mucin in these muco-epidermoid carcinomas varies considerably from tumour to tumour as well as in different areas of the same tumour. In 11 cases there were only small amounts of secretion. In 10 the amount of mucin was sufficiently abundant to be obvious in an ordinary haematooxylin-and-eosin-stained section. In general the amount of mucin and the size of the microcysts was greater in the growths of low grade malignancy.

The cell type in these carcinomas is transitional more often than squamous. In some tumours there is stratification in the middle of the clumps of cells with the formation of epithelial pearls but very little 


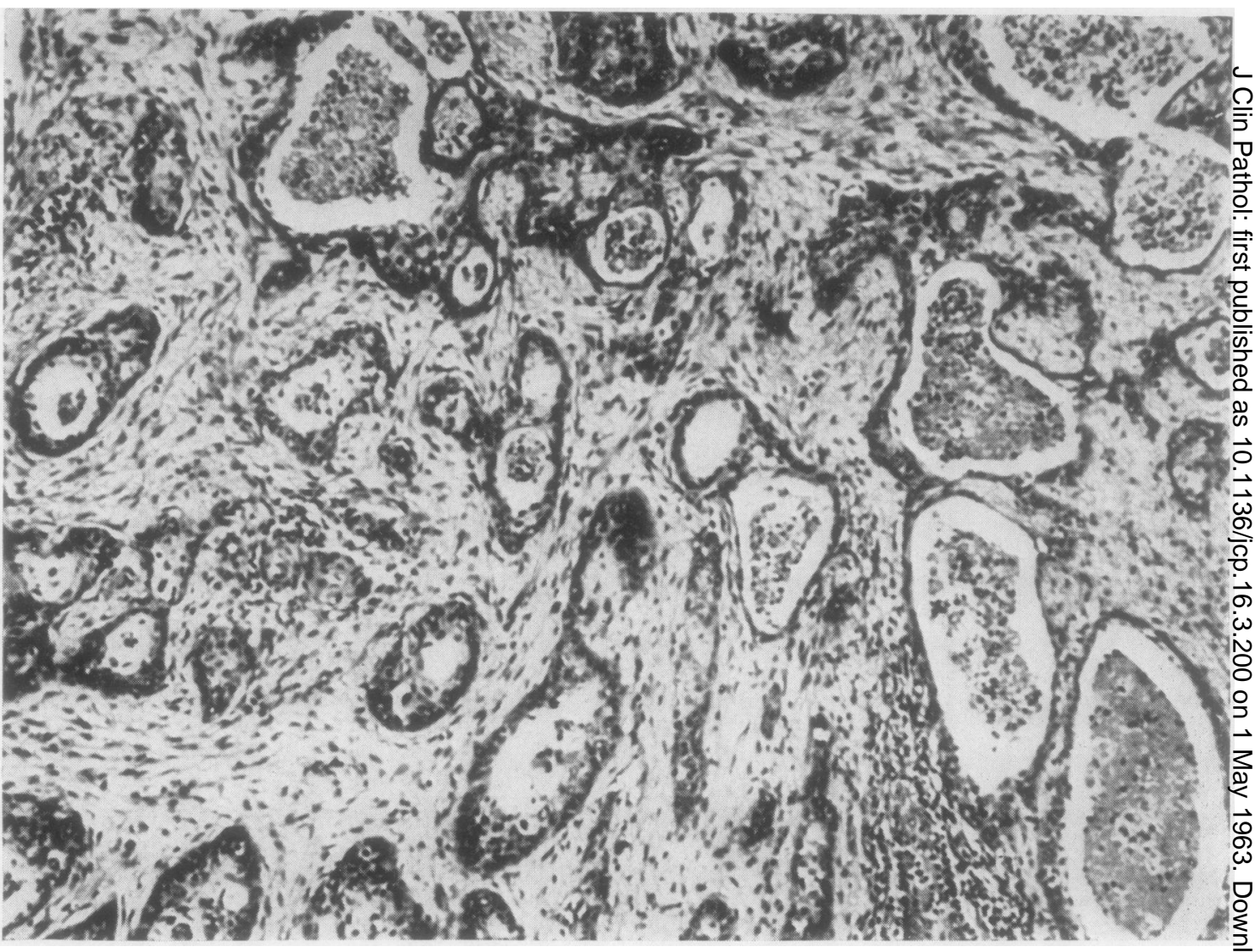

FIG. 2. Muco-epidermoid carcinoma of low-grade malignancy. Nearly all the clumps of tumour cells contain prominent microcysts filled with mucin and cell debris. Haematoxylin and mucicarmine $\times 100$.

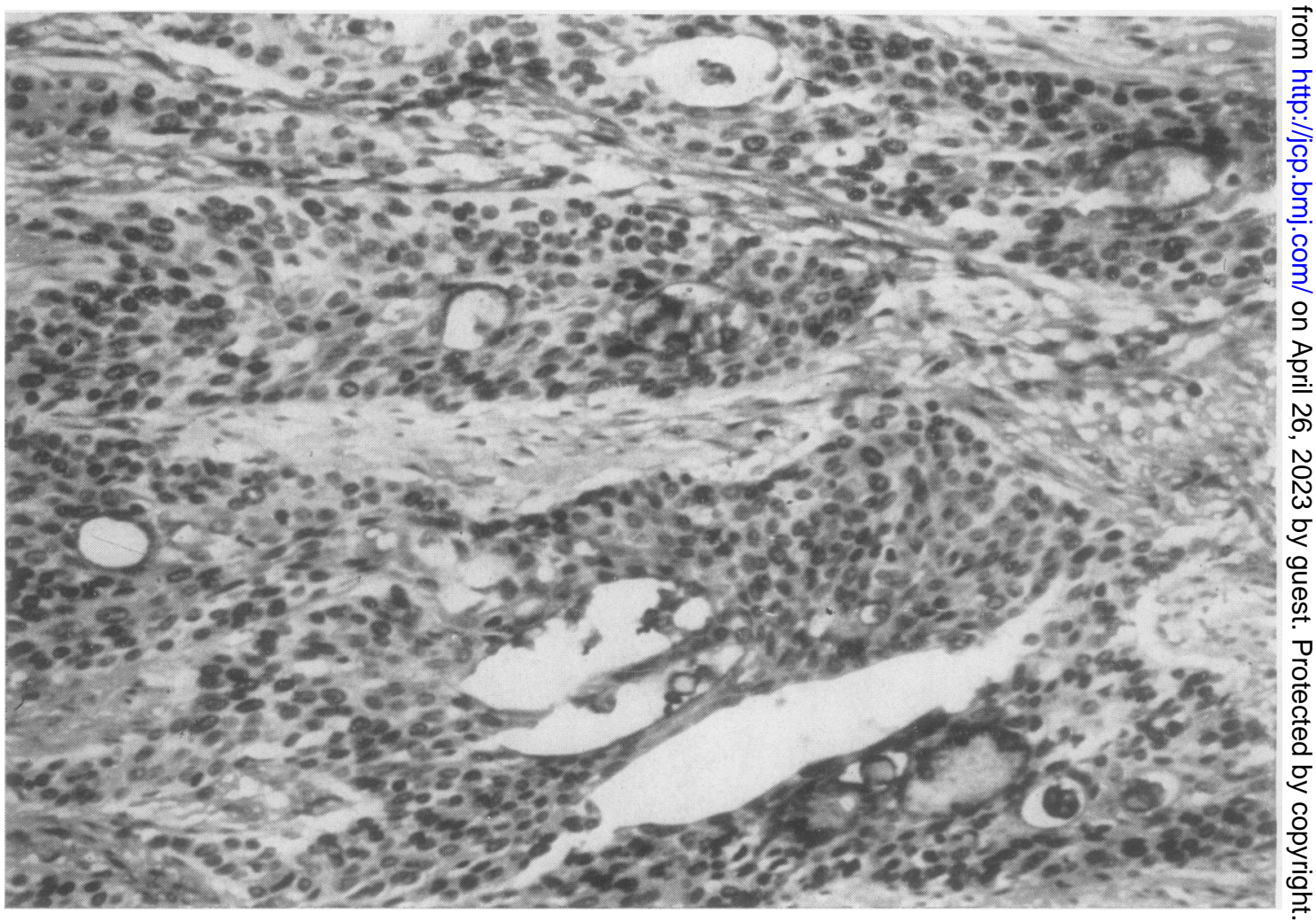

FIG. 3. Transitional cell carcinoma of anal canal containing many small microcysts and pools of mucin. Haematoxylin and alcian blue $\times 125$. 


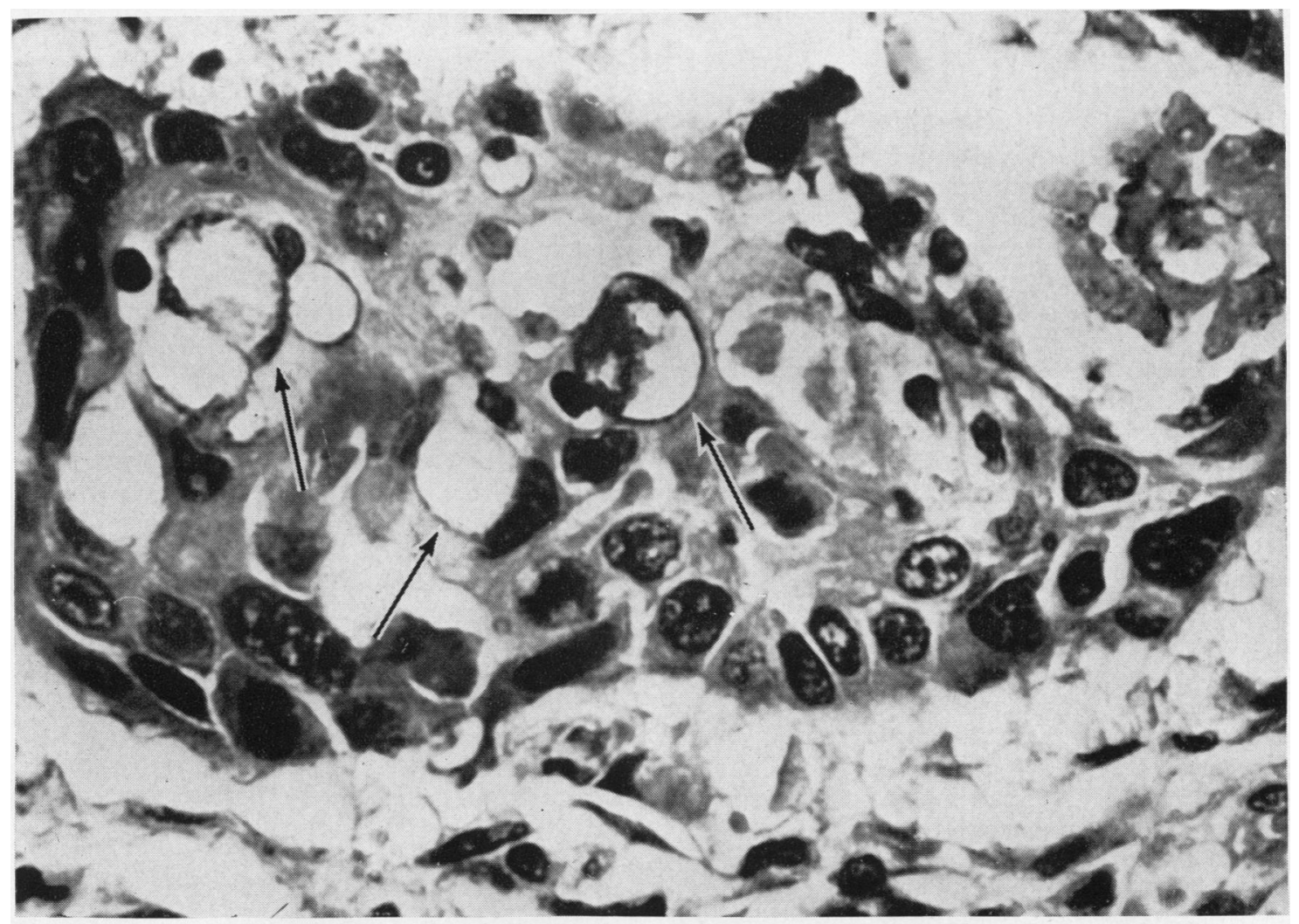

FIG. 4. Squamous carcinoma containing clumped mucus-secreting signet-ring cells (arrowed). Haematoxylin and alcian blue $\times 200$.

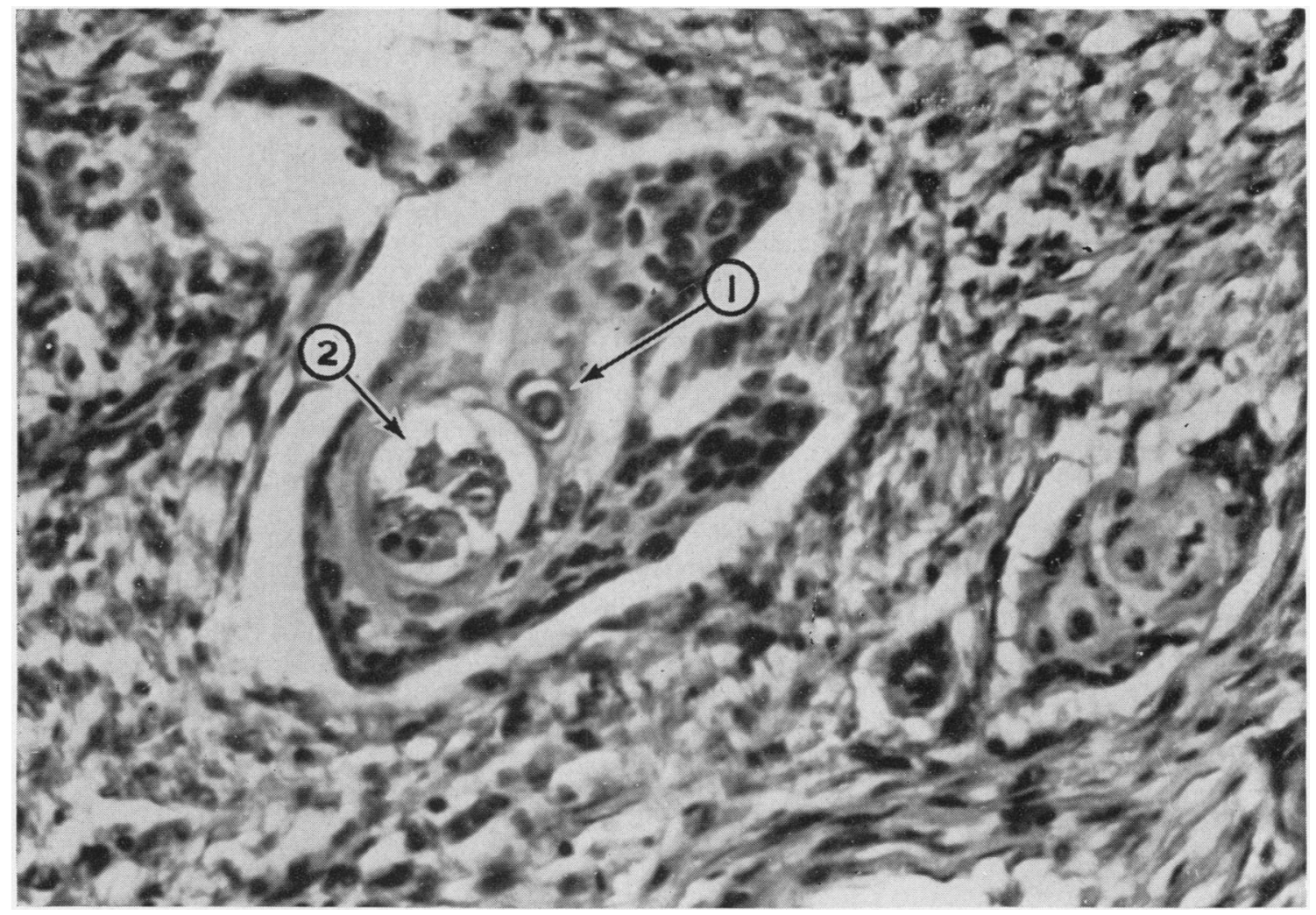

FIG. 5. Squamous carcinoma containing a signet-ring cell (arrowed 1) and some mixed keratin and mucin (arrowed 2). 
keratin. When keratin is present it may be mixed up with mucin giving the impression of a dual direction of differentiation (Fig. 5). No prickle cells could be found in any of these cases. This is to be expected, for prickle cells are very rarely present in squamous carcinomas of the anal canal whereas they are a regular feature of anal margin tumours. Similarly keratinization is a common feature of anal margin tumours and is exceptional in tumours of the anal canal, the majority of which are transitional in character. These differences in cell type and differentiation are a reflection of the normal histology of the epithelial lining of the anal canal and anal margin (Walls, 1958).

The histogenesis of the muco-epidermoid tumours reported here could not be clearly demonstrated as the tumour mass had largely destroyed the site of origin. However, in three cases there appeared to be continuity with the surface epithelium of the anal canal including the anal ducts, but only where they opened out on to the surface in the region of the anal crypts. This surface epithelium was mainly of the non-cornifying squamous and transitional type but continuity with stratified columnar epithelium could also be established. In none of these mucoepidermoid carcinomas was there any histological evidence of exclusive origin from the anal glands.

SPREAD There were no special features in the spread of these growths which distinguished them from the direction of spread of adenocarcinoma of the lower rectum or squamous cell carcinoma of the anal canal. Lymph node metastases to the superior haemorrhoidal group of lymphatic glands were present in 10 out of the 18 operation specimens. These showed the same histological appearances as the primary tumour in the anal canal. In seven patients inguinal gland involvement was also demonstrated. It has been shown elsewhere (Morson, 1960) that the direction of lymph node metastasis in anal canal carcinoma is about equally divided between the haemorrhoidal and inguinal glands.

\section{PROGNOSIS}

Fourteen of the 21 patients died from local recurrence or metastases within five years of surgical treatment.

\section{DISCUSSION}

It is not surprising that the anal canal should be a site where muco-epidermoid carcinoma is found or that these tumours should arise from that part of the canal just above the dentate line, because it is in this area that transitional types of epithelium between rectal mucosa and squamous mucous membrane are found. In his description of the epithelial lining of $\underline{\partial}$ the anal canal Walls (1958) has shown that there is great variation in the types of epithelium found in $\overline{\overrightarrow{0}}$ this 'transitional' or 'junctional' zone. Stratifiedo columnar epithelium is almost invariably present in the anal crypts and lining the anal glands. Transitional epithelium resembling bladder mucosa, non- $\stackrel{\mathbb{Q}}{\Omega}$ keratinizing squamous epithelium, and peculiar 'mixed' types of epithelium are regularly found. Sometimes mucus-secreting 'goblet' cells can be seen $\vec{\circ}$ within epithelium which is otherwise of standard $\overrightarrow{\vec{\omega}}$ squamous or transitional type (Morson, 1960). It $\stackrel{\omega}{\sigma}$ seems reasonable to postulate therefore that malignant change in the epithelial lining of the upper anal canal above the line of the valves should sometimes $\frac{\sigma}{\omega}$ give rise to a type of tumour the cells of which are predominantly transitional or squamous but include 8 some that retain the ability to secrete mucin.

Although evidence of histogenesis at a microscopic level in most of the cases reported here had $\bar{z}$ been destroyed, there were no reasons to suppose that the growths had not arisen from the surface $\vec{\varphi}$ epithelium of the anal canal. Kay (1954) and Close and Schwab (1955), however, have described mucoepidermoid tumours which they believed were derived from the anal glands. Willis (1960) also gives a brief description of a carcinoma of anal glands together with a photomicrograph of the tumour $\frac{D}{D}$

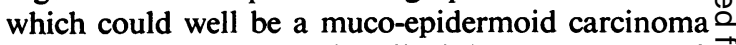
of the anal canal as described here. Berg et al. $\overline{\bar{O}}$ (1960) believe that the anal ducts are attractive as 3 sites of origin for cancers of this type. But in all these reports reliable evidence of an origin from anal glands is lacking.

The anal glands or ducts are extremely small, microscopic structures (Parks, 1961; Parks and 3 . Morson, 1962). They are lined by stratified columnar epithelium which gives a positive reaction for 3 epithelial mucin at its free margin. This epithelium $\mathrm{O}$ sometimes undergoes squamous or transitional cell metaplasia. There is no reason why carcinoma should on not sometimes arise from the anal ducts and have $a)$ muco-epidermoid structure. Bearing in mind, how- $\sigma$ ever, the embryology and histology of the lining $N$ epithelium of the upper anal canal, it would appearN more probable that most muco-epidermoid car- $\omega$ cinomas are derived from the surface epithelium. The upper anal canal is anyway an area from which most squamous and transitional cell carcinomas of the anal region are known to arise. Certainly there is no evidence from the study of the St. Mark's caseso that any of these muco-epidermoid tumours arose exclusively from the anal ducts. However, it is pos- $\frac{?}{\mathbb{D}}$ sible that these structures may play a minor role in $\frac{\varrho}{9}$ some cases for they are in continuity with the surface? 
epithelium. If the anal glands were ever an exclusive source of malignant disease the tumours would be mainly submucous in position. But the gross appearance of the growths reported here was ulcerative and did not in any way suggest an exclusive origin from intramural tissues of the anal canal.

The variability and instability of the epithelium lining the upper part of the anal canal is not sufficiently realized. It varies with the individual, age, and pathological conditions, such as haemorrhoids. Squamous metaplasia of the stratified columnar and transitional types of epithelium, in particular, is common, and this is the probable source of many squamous cell carcinomas of the anal canal. The fact that some of them have retained an ability to secrete mucin is only a reflection of the nature of the primordial tissue. It is believed that a similar histogenesis from a comparable squamo-glandular junction accounts for the presence of muco- epidermoid tumours in the cervical canal (Hamperl and Hellweg, 1957).

\section{REFERENCES}

Azzopardi, J. G., and Menzies, T. (1962). Brit. J. Surg., 49, 497. Berg, J. W., Lone, F., and Stearns, M. W. Jr. (1960). Cancer (Philad.), $13,914$.

Close, A. S., and Schwab, R. L. (1955). Ibid., 8, 979.

Dukes, C. E., and Bussey, H. J. R. (1958). Brit. J. Cancer, 12, 309.

Feyrter, F. (1956). Virchows. Arch. path. Anat., 328, 378.

Foote, F. W., and Frazell, F. L. (1954) Atlas of Tumour Pathology, Sections iv, fascicle ii. Armed Forces Institute of Pathology.

Hamperl, H., and Hellweg, G. (1957). Cancer (Philad.), 10, 1187.

Hellweg, G. (1957). Z. Krebsforsch., 61, 688.

Kay, S. (1954). Cancer (Philad.), 7, 359.

Morson, B. C. (1960). Proc. roy. Soc. Med., 53, 416.

Parks, A. G. (1961). Brit. med. J., 1, 463. and Morson, B. C. (1962). Proc. roy. Soc. Med., 55, 751.

Smith, A. G., Broadbent, T. R., and Zavaleta, A. A. (1954). Cancer (Philad.), 7, 224.

Stewart, F. W., Foote, F. W., and Becker, W.fF. (1945). Ann. Surg., $122,820$.

Walls, E. W. (1958). Brit. J. Surg., 45, 504.

Willis, R. A. (1960). Pathology of Tumours, 3rd ed. Butterworth, London. 